\title{
SELECTIONS GENERATING NEW TOPOLOGIES
}

\author{
Valentin Gutev and Artur Tomita
}

\begin{abstract}
Every (continuous) selection for the non-empty 2-point subsets of a space $X$ naturally defines an interval-like topology on $X$. In the present paper, we demonstrate that, for a second-countable zerodimensional space $X$, this topology may fail to be first-countable at some (or, even any) point of $X$. This settles some problems stated in [7].
\end{abstract}

\section{Introduction}

Let $X$ be a topological space, and let $\mathscr{F}(X)$ be the set of all nonempty closed subsets of $X$. Also, let $\mathscr{D} \subset \mathscr{F}(X)$. A map $f: \mathscr{D} \rightarrow X$ is a selection for $\mathscr{D}$ if $f(S) \in S$ for every $S \in \mathscr{D}$. A selection $f: \mathscr{D} \rightarrow X$ is continuous if it is continuous with respect to the relative Vietoris topology $\tau_{V}$ on $\mathscr{D}$. Let us recall that $\tau_{V}$ is generated by all collections of the form

$$
\langle\mathscr{V}\rangle=\{S \in \mathscr{F}(X): S \subset \bigcup \mathscr{V} \text { and } S \cap V \neq \varnothing, \text { whenever } V \in \mathscr{V}\},
$$

where $\mathscr{V}$ runs over the finite families of open subsets of $X$.

In the sequel, all spaces are assumed to be at least Hausdorff and infinite. In the present paper, we are interested in continuous selections for $\mathscr{D}$, when $\mathscr{D}$ is the family $\mathscr{F}_{2}(X)=\{S \in \mathscr{F}(X):|S| \leq 2\}$. In this case, a selection $f: \mathscr{F}_{2}(X) \rightarrow X$ is usually called a weak selection for $X$.

Every weak selection $f$ for $X$ defines an order-like relation $\preceq_{f}$ on $X$ (see [10]) by letting that $x \preceq_{f} y$ iff $f(\{x, y\})=x$. For convenience, we write that $x \prec_{f} y$ if $x \preceq_{f} y$ and $x \neq y$. We note that the relation " $\preceq_{f}$ " may fail to be transitive (see, for instance, [4, Proposition 2.2]). Nevertheless, to every continuous weak selection $f$ for $X$ we may associate a

2000 Mathematics Subject Classification. 54B20, 54C65.

Key words. Hyperspace topology, Vietoris topology, continuous selection.

The second author would like to thank UMALCA for the support to cover a part of the air fare expenses for his visit to UNAM, Campus Morelia, Mexico in December 2004, where the research was partially conducted. 
topology $\mathscr{T}_{f}$ on $X$ generated by all "open $f$-intervals" $\left\{y \in X: y \prec_{f} x\right\}$ and $\left\{y \in X: x \prec_{f} y\right\}, x \in X$. According to [10, Lemma 7.2] (see, also, $[\mathbf{4}$, Lemma 3.3]), these " $f$-intervals" are always open in the original topology of $X$. Hence, $\mathscr{T}_{f}$ is a coarser topology on $X$, and, consequently, it is the original topology on $X$ provided $X$ is compact. In fact, by [11, Theorem 1.1], for a compact space $X$ the topology $\mathscr{T}_{f}$ coincides with the open interval topology on $X$ generated by a linear ordering on $X$ (i.e., $\mathscr{T}_{f}$ is an order topology on $X$ ). According to [10, Lemma 7.2], $\mathscr{T}_{f}$ is also an order topology on $X$ provided $X$ is connected. Finally, by [12, Theorem 4 and Remark 16], $\mathscr{T}_{f}$ coincides with the original topology on $X$ provided $X$ is connected and locally connected.

Some further properties of this topology were studied in [4], [7]. For instance, by $\left[\mathbf{7}\right.$, Corollary 2.3], $\mathscr{T}_{f}$ is always a regular topology on $X$. On the other hand, by $\left[\mathbf{7}\right.$, Corollary 2.4], $\mathscr{T}_{f}$ is the usual Euclidean topology on the rational numbers $\mathbb{Q}$, whenever $f$ is a continuous weak selection for $\mathbb{Q}$. Hence, it become quite natural to study this topology on the irrational numbers $\mathbb{P}$ which is an uncountable, second countable, zero-dimensional space.

We are now ready to state the main purpose of this paper. Namely, in this paper, we show that every uncountable, non-compact, secondcountable, zero-dimensional space $X$ has a continuous weak selection $f$ such that $\mathscr{T}_{f}$ is not first-countable at some point of $X$, see Theorem 4.1. In the same theorem, we also demonstrate that $\mathscr{T}_{f}$ is not first-countable at any point of $X$ provided $X$ has an infinite pairwise disjoint cover consisting of uncountable open sets. Thus, in particular, there exists a continuous weak selection $f$ for the irrational numbers $\mathbb{P}$ such that $\mathscr{T}_{f}$ is not first-countable at any point of $\mathbb{P}$ (Corollary 4.2), which provides a negative answer to $[\mathbf{7}$, Question 2], and a positive one to $[\mathbf{7}$, Question 3]. Another interesting consequence is that an uncountable metrizable space $X$, with a covering $\operatorname{dimension} \operatorname{dim}(X)=0$, is compact if and only if $\mathscr{T}_{f}$ is second-countable for every continuous weak selection $f$ on $X$ (see Corollary 4.4). For other applications, we refer the interested reader to Sections 4 and 5 of the paper.

A preparation for the proof of Theorem 4.1 is given in Sections 2 and 3, while its proof will be finally accomplished in Section 4. A part of this preparation is based on a criterion for the existence of continuous weak selections (see Theorem 5.1), which is analogous to a result of Eilenberg on orderability [1]. It has a list of interesting independent consequences (see Section 5). 
In conclusion, the second author would like to express his best gratitude to Professor Salvador García-Ferreira for his support and hospitality, and for discussing some questions related to this research.

\section{A relation generated by weak selections}

Let $X$ be a set, and let $E \subset X \times X$ be a relation on $X$. As usual, we write $x E y$ to denote that $(x, y) \in E$. Let us recall that a relation $E$ on $X$ is anti-symmetric if $x E y$ and $y E x$ implies $x=y$. Following [7], we say that an anti-symmetric relation $E$ on $X$ is a selection relation if $x E y$ or $y E x$ for every $x, y \in X$. Let us emphasize that, in this terminology, a relation $E$ on $X$ is a linear order on $X$ if $E$ is a selection relation which is also transitive (i.e., $x E y$ and $y E z$ implies $x E z$ ).

It should be mentioned that the set of all possible weak selections for $X$ corresponds precisely to all possible selection relations on $X$. Namely, any selection relation $E$ on $X$ defines a weak selection $f_{E}$ by letting $f_{E}(\{x, y\})=x$ iff $x E y$. On the other hand, if $f$ is a weak selection for $X$, then the order-like relation $\preceq_{f}$ generated by $f$ is a selection relation. In the sequel, we will refer to $\preceq_{f}$ as a selection relation.

In the present section, we are interested in a natural extension of such relations to the subsets of $X$. Following [3], for a selection relation " $\preceq$ " and (not necessarily non-empty) subsets $B, C \subset X$, we shall write that $B \preceq C$ (respectively, $B \prec C$ ) if $y \preceq z$ (respectively, $y \prec z$ ) for every $y \in B$ and $z \in C$. Obviously, $B \prec C$ implies $B \cap C=\varnothing$.

In these terms, we have the following simple criterion for continuity in $\mathscr{F}_{2}(X)$ which is, in fact, [4, Theorem 3.1].

Proposition $2.1([4])$. Let $X$ be a space, $f$ be a weak selection for $X$, and let " $\preceq_{f}$ " be the selection relation generated by $f$. Also, let $x, y \in X$ be such that $x \prec_{f} y$. Then, $f$ is continuous at $\{x, y\}$ if and only if there are open sets $U$ and $V$ such that $x \in U, y \in V$, and $U \prec_{f} V$.

On the other hand, we have the following property of weak selections. It was implicitly used in several papers and summarized in $[\mathbf{6}$, Proposition 4.1].

Proposition 2.2. Let $X$ be a space, and let $f$ be a weak selection. Then, $f$ is continuous on the singletons of $X$.

Motivated by Propositions 2.1 and 2.2, we may consider only the subset

$$
[X]^{2}=\left\{S \in \mathscr{F}_{2}(X):|S|=2\right\},
$$


which will play a crucial role in this paper. In fact, we will make no difference between weak selections $f: \mathscr{F}_{2}(X) \rightarrow X$ and weak selections $f:[X]^{2} \rightarrow X$.

The following simple observation about special weak selections will be also useful.

Proposition 2.3. Let $X$ be a space which has a continuous weak selection, and an infinite pairwise disjoint cover $\mathscr{V}$ consisting of non-empty open subsets. Then, there exists a continuous weak selection $g:[X]^{2} \rightarrow$ $X$ such that $\mathscr{V}$ is an unbounded well-ordered set with respect to the selection relation generated by $g$.

Proof: Let $f:[X]^{2} \rightarrow X$ be a continuous weak selection. Also, let $h: \delta \rightarrow$ $\mathscr{V}$ be a one-to-one map, where $\delta=|\mathscr{V}|$. Then, for every $x \in X$, let $\alpha(x)<\delta$ be such that $x \in h(\alpha(x))$. Finally, define $g:[X]^{2} \rightarrow X$ by letting for distinct points $x, y \in X$ that $g(\{x, y\})=x$ if $\alpha(x)<\alpha(y)$, and $g(\{x, y\})=f(\{x, y\})$ if $\alpha(x)=\alpha(y)$. Clearly, $g$ is continuous because so is $f$, and $\mathscr{V}$ is a discrete open cover of $X$. On the other hand, by the definition of $g$, the selection relation $\preceq_{g}$ defines the same order on $\mathscr{V}$ as that one of the infinite cardinal $\delta$. Hence, $\mathscr{V}$ is unbounded and wellordered with respect to $\preceq_{g}$.

We conclude this section with some properties of the topology generated by weak selections. Suppose that $f$ is a weak selection for $X$, and $\preceq_{f}$ is the selection relation generated by $f$. For every $x \in X$, we consider the corresponding "open $f$-intervals"

$$
\mathbb{I}_{f}(x, \infty)=\left\{y \in X: x \prec_{f} y\right\}, \quad \text { and } \quad \mathbb{I}_{f}(\infty, x)=\left\{y \in X: y \prec_{f} x\right\} .
$$

Also, for convenience, we let

$$
\mathscr{I}_{f}(X)=\left\{\mathbb{I}_{f}(\infty, x), \mathbb{I}_{f}(x, \infty): x \in X\right\} .
$$

In these terms, the topology $\mathscr{T}_{f}$ is generated by all finite intersections of members of $\mathscr{I}_{f}(X)$. This is the place to recall that, in general, the relation $\preceq_{f}$ is not transitive. Hence, we may have points $x, y, z \in X$ which generate an infinite "monotone" sequence

$$
\cdots \prec_{f} x \prec_{f} y \prec_{f} z \prec_{f} x \prec_{f} \cdots
$$

In particular, for such points, we also have that

$$
\left\{t \in X: x \prec_{f} t \prec_{f} y\right\} \neq \varnothing \neq\left\{t \in X: y \prec_{f} t \prec_{f} x\right\} .
$$

Motivated by this, for every $a, b \in X$ we will associate the set

$$
\mathbb{I}_{f}(a, b)=\mathbb{I}_{f}(a, \infty) \cap \mathbb{I}_{f}(\infty, b)=\left\{x \in X: a \prec_{f} x \prec_{f} b\right\} .
$$


However, we don't require that $a \prec_{f} b$. Hence, both $f$-intervals $\mathbb{I}_{f}(a, b)$ and $\mathbb{I}_{f}(b, a)$ make sense, and could be non-empty.

In what follows, we shall say that a point $x \in X$ is an $f$-cutting point if there are points $a, b \in X$, with $x \in \mathbb{I}_{f}(a, b)$. Otherwise, we shall say that $x$ is an $f$-extreme point of $X$. Clearly, $X$ may have at most two $f$-extreme points, which could be different for different selections $f$.

Proposition 2.4. Let $X$ be a space, $f$ be a weak selection for $X$, and let $A, B \subset X$ be non-empty subsets such that

$$
\mathbb{I}_{f}(A, B)=\bigcap\left\{\mathbb{I}_{f}(a, b):(a, b) \in A \times B\right\} \neq \varnothing .
$$

Then, $A \cap B=\varnothing$. In particular, if $x \in X$ is an $f$-cutting point and $U \in \mathscr{T}_{f}$, then $x \in U$ if and only if there are non-empty finite disjoint subsets $A, B \subset X$, with $x \in \mathbb{I}_{f}(A, B) \subset U$.

Proof: The first part of this statement follows from the fact that $\mathbb{I}_{f}(z, z)=$ $\varnothing$ for every $z \in X$. As for the second part, by the definition of $\mathscr{T}_{f}, x \in U$ if and only if there is a finite set $\mathscr{K} \subset \mathscr{I}_{f}(X)$, with $x \in \bigcap \mathscr{K} \subset U$. On the other hand, $x \in \mathbb{I}_{f}(a, b)$ for some $a, b \in X$, because $x$ is an $f$-cutting point. Let $A_{0}=\left\{y \in X: \mathbb{I}_{f}(y, \infty) \in \mathscr{K}\right\}$ and $B_{0}=\left\{z \in X: \mathbb{I}_{f}(\infty, z) \in\right.$ $\mathscr{K}\}$. Then, $A=A_{0} \cup\{a\}$ and $B=B_{0} \cup\{b\}$ are as required.

\section{A condition for continuity of weak selections}

Lemma 3.1. Let $X$ be a space, $f:[X]^{2} \rightarrow X$ be a selection, and let $\preceq_{f}$ be the selection relation generated by $f$. Then, $f$ is continuous if and only if the set $\mathscr{L}=\left\{(x, y) \in X^{2}: x \prec_{f} y\right\}$ is open in $X^{2}$. In particular, if $f$ is continuous, then the map $h: \mathscr{L} \rightarrow[X]^{2}$, defined by $h((x, y))=\{x, y\},(x, y) \in \mathscr{L}$, is a homeomorphism.

Proof: Take distinct points $x, y \in X$ such that $(x, y) \in \mathscr{L}$, i.e. $x \prec_{f} y$. Then, by Proposition 2.1, $f$ is continuous at $\{x, y\}$ if and only if there are open sets $U, V \subset X$ such that $x \in U, y \in V$, and $U \prec_{f} V$. According to the definition of $\mathscr{L}$, this implies that $f$ is continuous at $\{x, y\}$ if and only if there are disjoint open subsets $U, V \subset X$ such that $(x, y) \in U \times V \subset \mathscr{L}$. In particular, if $f$ is continuous, then the map $h$ is a continuous open bijection which completes the proof.

Lemma 3.1 suggests a natural construction of continuous weak selections. To this end, for a subset $Z \subset X^{2}$, let us agree to say that $\pi: Z \rightarrow X$ is a projection if $\pi((x, y)) \in\{x, y\}$ for every $(x, y) \in Z$. Then, whenever $Z \subset X^{2}$, we have always two standard continuous projections $\pi_{i}: Z \rightarrow X, i=0,1$, defined by $\pi_{0}((x, y))=x$ and $\pi_{1}((x, y))=y$, 
$(x, y) \in Z$. Here is another example of continuous projections, which will play an important role in the next section.

Example 3.2. Let $X$ be a space, $Z \subset X^{2}, \mathscr{U}$ be a discrete open cover of $Z$, and let $\xi: \mathscr{U} \rightarrow 2=\{0,1\}$ be an arbitrary map. Define a map $\pi: Z \rightarrow X$ by letting for $(x, y) \in Z$ that $\pi((x, y))=\pi_{\xi(U)}((x, y))$ if $(x, y) \in U \in \mathscr{U}$. Then, $\pi$ is a continuous projection such that $\pi \uparrow U=$ $\pi_{\xi(U)}\lceil U, U \in \mathscr{U}$.

Proof: Follows from the fact that $\mathscr{U}$ is a discrete open cover of $Z$.

According to Lemma 3.1, we now have the following immediate consequence. It provides a possible way to construct continuous weak selections from given ones.

Corollary 3.3. Let $X$ be a space, $f:[X]^{2} \rightarrow X$ be a continuous selection, and let $\mathscr{L}$ and $h: \mathscr{L} \rightarrow[X]^{2}$ be as in Lemma 3.1. Also, let $\pi: \mathscr{L} \rightarrow X$ be a continuous projection. Then, $g=\pi \circ h^{-1}:[X]^{2} \rightarrow X$ is a continuous selection.

\section{A construction of continuous weak selections}

Theorem 4.1. Let $X$ be an uncountable, non-compact, second-countable, zero-dimensional space. Then, $X$ has a continuous weak selection $f$ such that $\mathscr{T}_{f}$ is not first-countable at some point of $X$. If, moreover, $X$ has an infinite pairwise disjoint open cover consisting of uncountable sets, then $X$ has a continuous weak selection $f$ such that $\mathscr{T}_{f}$ is not firstcountable at any point of $X$.

Proof: First of all, let us observe that $X$ is regular because it has a base of clopen sets. Hence, by the Urysohn's metrization theorem [13] (see, also, [2]), $X$ is metrizable. Also, $\operatorname{dim}(X)=0[\mathbf{1 4}]$ (see, also, $[\mathbf{2}]$ ) because $X$ is a Lindelöf space being regular and second-countable.

In case $X$ has an infinite cover consisting of pairwise disjoint uncountable open sets, we let this cover to be $\mathscr{V}$. Otherwise, let us observe that $X$ has an infinite cover $\mathscr{V}$ consisting of non-empty pairwise disjoint open sets such that at least three members of $\mathscr{V}$ are uncountable. To this end, let $Z$ be the set of all points $x \in X$ such that $x$ has a local base consisting of uncountable open sets. Then, $Z$ must be closed because every neighbourhood of a point $z \in \bar{Z}$ will contain a point of $Z$. In this case, $X \backslash Z$ must be countable. Namely, take a countable base $\mathscr{O}$ for the topology of $X$, and then observe that $X \backslash Z=\bigcup\{O \in \mathscr{O}:|O| \leq \omega\}$. Since $X$ is uncountable, $Z$ must be also uncountable and, in particular, 
infinite. Thus, using that $\operatorname{dim}(X)=0$, we can take $\mathscr{V}$ to be an infinite cover of $X$ consisting of non-empty pairwise disjoint open sets, with $|\{V \in \mathscr{V}: V \cap Z \neq \varnothing\}| \geq 3$.

Having already constructed the cover $\mathscr{V}$, let $\mathscr{B}$ be a countable base for the topology of $X$ consisting of non-empty clopen subsets such that $\mathscr{B}$ is a refinement of $\mathscr{V}$. In what follows, we will use $\mathscr{D}(\mathscr{B})$ to denote the set of all non-empty subsets $\mathscr{W} \subset \mathscr{B}$ which are finite and pairwise disjoint. Next, for every $\mathscr{W} \in \mathscr{D}(\mathscr{B})$, let $2^{\mathscr{W}}$ be the set of all maps $\mu: \mathscr{W} \rightarrow 2=\{0,1\}$. Also, we let

$$
\mathscr{M}_{0}=\bigcup\left\{2^{\mathscr{W}}: \mathscr{W} \in \mathscr{D}(\mathscr{B})\right\} .
$$

Finally, for every $\mu \in \mathscr{M}_{0}$, we let $\operatorname{Dom}(\mu)$ to be the domain of $\mu$, which is clearly a non-empty finite and pairwise disjoint subset of $\mathscr{B}$.

Note that $X$ has a continuous weak selection, because it is a subset of the Cantor set [14] (see, also, [2]) being a regular space with a countable clopen base. Hence, by Proposition $2.3, X$ has a continuous weak selection $g$ such that $\mathscr{V}$ is an unbounded well-ordered set with respect to the selection relation " $\preceq_{g}$ " generated by $g$. Now, let $\mathscr{V}^{*}=\{V \in \mathscr{V}:|V|>\omega\}$, which, by construction, has the property that $\left|\mathscr{V}^{*}\right| \geq 3$. Next, let $V^{*}=\min _{\preceq_{g}} \mathscr{V}^{*}$, and then take $x^{*} \in V^{*}$ and $W^{*} \in \mathscr{B}$ to be such that $x^{*} \in W^{*} \subset V^{*}$. Finally, define

$$
\mu_{i}^{*}:\left\{W^{*}\right\} \rightarrow 2, i=0,1, \quad \text { by } \mu_{i}^{*}\left(W^{*}\right)=1-i .
$$

Thus, we get two different elements $\mu_{0}^{*}, \mu_{1}^{*} \in \mathscr{M}_{0}$, so we let

$\alpha\left(\mu_{0}^{*}\right)=\min _{\preceq_{g}}\left(\mathscr{V}^{*} \backslash\left\{V^{*}\right\}\right) \quad$ and $\quad \alpha\left(\mu_{1}^{*}\right)=\min _{\preceq_{g}}\left(\mathscr{V}^{*} \backslash\left\{V^{*} \cup \alpha\left(\mu_{0}^{*}\right)\right\}\right)$.

For later use, let us observe that

$$
\begin{aligned}
W^{*} \prec_{g} \alpha\left(\mu_{0}^{*}\right) & \prec_{g} \alpha\left(\mu_{1}^{*}\right), \\
& \text { while both } \alpha\left(\mu_{0}^{*}\right) \text { and } \alpha\left(\mu_{1}^{*}\right) \text { are uncountable. }
\end{aligned}
$$

Now, we are going to extend the map $\alpha:\left\{\mu_{0}^{*}, \mu_{1}^{*}\right\} \rightarrow \mathscr{V}$ to an injective map $\alpha: \mathscr{M}_{0} \rightarrow \mathscr{V}$ such that, for every $\mu \in \mathscr{M}_{0}$,

$$
W \prec_{g} \alpha(\mu), \quad \text { whenever } W \in \operatorname{Dom}(\mu) .
$$

This can be done by transfinite induction because $\mathscr{M}_{0}$ is countable, while $\mathscr{V}$ is infinite, hence $\left|\mathscr{M}_{0}\right| \leq|\mathscr{V}|$. Namely, take a well-ordering $\ll$ on $\mathscr{M}_{0}$ as that of the first infinite ordinal $\omega$ such that $\mu_{0}^{*}=\min _{\ll} \mathscr{M}_{0}$ and $\mu_{1}^{*}=\min _{\ll}\left(\mathscr{M}_{0} \backslash\left\{\mu_{0}^{*}\right\}\right)$. Next, suppose that $\alpha(\nu)$ has been already defined for every $\nu \ll \mu$ and some $\mu \in \mathscr{M}_{0}$, with $\mu \gg \mu_{1}^{*}$. Then,

$$
\mathscr{V}_{\mu}=\{\alpha(\nu): \nu \ll \mu\} \cup\{V \in \mathscr{V}: W \subset V \text { for some } W \in \operatorname{Dom}(\mu)\},
$$


is a non-empty finite subset of $\mathscr{V}$, while $\mathscr{V}$ is unbounded. Hence the set

$$
\mathscr{V}^{\mu}=\left\{V \in \mathscr{V}: \max _{\preceq_{g}} \mathscr{V}_{\mu} \prec_{g} V\right\}
$$

is also non-empty. So, we may define $\alpha(\mu)=\min _{\preceq_{g}} \mathscr{V}^{\mu}$, which completes the construction.

Now, for convenience, we let $\vartheta:\{X\} \rightarrow 2=\{0,1\}$ to be the map $\vartheta(X)=0$, and $\mathscr{M}=\mathscr{M}_{0} \cup\{\vartheta\}$. Also, we let $A_{0}=\bigcup\left(\mathscr{V} \backslash \alpha\left(\mathscr{M}_{0}\right)\right)$, $\alpha(\vartheta)=A_{0}$, and $\mathscr{A}=\alpha\left(\mathscr{M}_{0}\right) \cup\left\{A_{0}\right\}$. Thus, we get a discrete partition $\mathscr{A}$ of $X$ and a one-to-one map $\alpha: \mathscr{M} \rightarrow \mathscr{A}$ such that (4.3) holds for every $\mu \in \mathscr{M}_{0}$. Keeping in mind this, we are going to construct a discrete open partition $\mathscr{U}=\left\{U_{\alpha(\mu)}^{i}: \mu \in \mathscr{M}\right.$ and $\left.i=0,1\right\}$ of the set $\mathscr{L}=\{(x, y) \in$ $\left.X^{2}: x \prec_{g} y\right\}$. To this end, to every subset $F \subset X$ and every $k \in 2$, we associate another subset $\mathscr{S}(k, F)$ defined by

$$
\mathscr{S}(k, F)= \begin{cases}X \backslash F & \text { if } k=0 \\ F & \text { if } k=1 .\end{cases}
$$

Then, for every $\mu \in \mathscr{M}$, we define

$$
U_{\alpha(\mu)}^{0}=\mathscr{L} \cap(\bigcup\{\mathscr{S}(1-\mu(W), W) \times \alpha(\mu): W \in \operatorname{Dom}(\mu)\}),
$$

and

$$
U_{\alpha(\mu)}^{1}=\mathscr{L} \cap(\bigcup\{\mathscr{S}(\mu(W), W) \times \alpha(\mu): W \in \operatorname{Dom}(\mu)\}) .
$$

It is easy to observe that $U_{\alpha(\mu)}^{0} \cup U_{\alpha(\mu)}^{1}=\mathscr{L} \cap(X \times \alpha(\mu))$, hence $\mathscr{U}$ is a partition of $\mathscr{L}$. Also, $\mathscr{U}$ is defined only by products of clopen sets, hence it is clopen as well.

Finally, we define a map $\xi: \mathscr{U} \rightarrow 2$ by letting $\xi(U)=i$ if $U=U_{\alpha(\mu)}^{i}$ for some $\mu \in \mathscr{M}$. Thus, by Example 3.2, we get a continuous projection $\pi: \mathscr{L} \rightarrow X$, with $\pi \uparrow U_{\alpha(\mu)}^{i}=\pi_{i} \uparrow U_{\alpha(\mu)}^{i}, \mu \in \mathscr{M}$ and $i=0,1$. Hence, by Corollary 3.3, $f=\pi \circ h^{-1}$ is a continuous selection for $[X]^{2}$. In what follows, let " $\preceq_{f}$ " be the selection relation generated by $f$.

We are going to show that $f$ is as required. To prepare for this, take $\mu \in \mathscr{M}_{0}, W \in \operatorname{Dom}(\mu)$ and $(x, y) \in W \times \alpha(\mu)$, and let us observe that

$$
f(\{x, y\})= \begin{cases}x & \text { if } \mu(W)=0, \\ y & \text { if } \mu(W)=1 .\end{cases}
$$

Indeed, by (4.3), we have $W \times \alpha(\mu) \subset \mathscr{L}$. If $\mu(W)=0$, then, by (4.4) and (4.5),

$$
\mathscr{S}(1-\mu(W), W) \times \alpha(\mu)=\mathscr{S}(1, W) \times \alpha(\mu)=W \times \alpha(\mu) \subset U_{\alpha(\mu)}^{0} .
$$


So $(x, y) \in U_{\alpha(\mu)}^{0}$, which implies that $f(\{x, y\})=\pi_{0}((x, y))=x$. If $\mu(W)=1$, then, in the same way, by (4.4) and (4.6),

$$
\mathscr{S}(\mu(W), W) \times \alpha(\mu)=\mathscr{S}(1, W) \times \alpha(\mu)=W \times \alpha(\mu) \subset U_{\alpha(\mu)}^{1},
$$

and therefore $f(\{x, y\})=\pi_{1}((x, y))=y$.

Now, take $y, z \in X$ and non-empty finite disjoint subsets $A, B \subset X$. We have the following crucial property of the topology $\mathscr{T}_{f}$.

$$
\{y, z\} \cap(A \cup B)=\varnothing \quad \text { implies } \quad \mathbb{I}_{f}(A, B) \backslash \mathbb{I}_{f}(y, z) \neq \varnothing .
$$

Indeed, consider the finite set $K=A \cup B \cup\{y, z\}$, and then take a pairwise disjoint family $\mathscr{W}=\left\{W_{x}: x \in K\right\} \subset \mathscr{B}$ such that $x \in W_{x}$ for every $x \in K$. Next, define $\mu: \mathscr{W} \rightarrow 2$ by letting for $x \in K$ that $\mu\left(W_{x}\right)=0$ if $x \in A$ or $x=z$, and $\mu\left(W_{x}\right)=1$ otherwise. Thus, we get a particular element $\mu$ of $\mathscr{M}_{0}$. Take a point $e \in \alpha(\mu)$, and let us observe that, by the definition of $\mu$ and (4.7), $x \prec_{f} e$ if $x \in A$ or $x=z$, and $e \prec_{f} x$ if $x \in B$ or $x=y$. That is, $e \in \mathbb{I}_{f}(A, B)$, but $e \notin \mathbb{I}_{f}(y, z)$.

We are finally ready to show that the selection $f$ is as required by showing that each point of $X$ is an $f$-cutting point. Take a point $x \in X$, and let $W \in \mathscr{B}$ be such that $x \in W$. Just like in (4.1), define $\mu_{i}:\{W\} \rightarrow 2$, $i=0,1$, by $\mu_{i}(W)=1-i$. Thus, we get two different elements $\mu_{0}, \mu_{1} \in \mathscr{M}_{0}$, so $\alpha\left(\mu_{0}\right) \neq \alpha\left(\mu_{1}\right)$. Then,

$$
x \in \mathbb{I}_{f}\left(y_{0}, y_{1}\right), \quad \text { whenever }\left(y_{0}, y_{1}\right) \in \alpha\left(\mu_{0}\right) \times \alpha\left(\mu_{1}\right) .
$$

Indeed, by (4.7), $\mu_{0}(W)=1$ implies $f\left(\left\{x, y_{0}\right\}\right)=y_{0}$ because $\left(x, y_{0}\right) \in$ $W \times \alpha\left(\mu_{0}\right)$, while $\mu_{1}(W)=0$ implies $f\left(\left\{x, y_{1}\right\}\right)=x$ because $\left(x, y_{1}\right) \in$ $W \times \alpha\left(\mu_{1}\right)$. This completes the verification of (4.9). In fact, it also implies that $\mathscr{T}_{f}$ is not first-countable at $x \in X$ if both $\alpha\left(\mu_{0}\right)$ and $\alpha\left(\mu_{1}\right)$ are uncountable. Namely, suppose if possible that $\mathscr{T}_{f}$ is first-countable at $x \in X$, but $\alpha\left(\mu_{0}\right)$ and $\alpha\left(\mu_{1}\right)$ are uncountable. Then, by Proposition 2.4, there is a countable set $E(x) \subset X$ such that for every $\mathscr{T}_{f}$-neighbourhood $U$ of $x$ there are non-empty finite disjoint subsets $A, B \subset E(x)$, with $x \in \mathbb{I}_{f}(A, B) \subset U$. On the other hand, there are points $y_{i} \in$ $\alpha\left(\mu_{i}\right) \backslash E(x), i=0,1$, because both $\alpha\left(\mu_{0}\right)$ and $\alpha\left(\mu_{1}\right)$ are uncountable. However, by (4.9), this implies that $x \in \mathbb{I}_{f}\left(y_{0}, y_{1}\right)$, while, by (4.8), it implies that $\mathbb{I}_{f}(A, B) \backslash \mathbb{I}_{f}\left(y_{0}, y_{1}\right) \neq \varnothing$ for every two non-empty finite disjoint subsets $A, B \subset E(x)$. The contradiction so obtained implies that $\mathscr{T}_{f}$ is not first-countable at $x$. In particular, by (4.2), it now implies that $\mathscr{T}_{f}$ is not first-countable at the point $x^{*}$ selected at the beginning of this proof. Finally, if each element of $\mathscr{V}$ is uncountable, then $\mathscr{T}_{f}$ will be not first-countable at any point of $X$, which completes the proof. 
The following is an immediate consequence of Theorem 4.1 which provides a negative answer to [7, Question 2], and a positive one to [7, Question 3].

Corollary 4.2. Let $\mathbb{P}$ be the set of the irrational numbers endowed with the usual Euclidean topology. Then, $\mathbb{P}$ has a continuous weak selection $f$ such that $\mathscr{T}_{f}$ is not first-countable at any point of $\mathbb{P}$.

Here is another interesting consequence.

Corollary 4.3. Let $\mathfrak{C}$ be the Cantor set, and let $p \in \mathfrak{C}$. Then, $X=$ $\mathfrak{C} \backslash\{p\}$ has a continuous weak selection $f$ such that $\mathscr{T}_{f}$ is not firstcountable at any point of $X$. In particular, $f$ cannot be extended to a continuous weak selection for $\mathfrak{C}$.

As it was mentioned in the Introduction, if $(X, \mathscr{T})$ is compact, then $\mathscr{T}_{f}=\mathscr{T}$ for every continuous weak selection $f$ for $X$. The same is true if $(X, \mathscr{T})$ is connected and locally connected, but we don't know if this holds for other classes of spaces (see [7, Question 4]). Related to this, we have the following further consequence of Theorem 4.1.

Corollary 4.4. Let $X$ be an uncountable metrizable space $X$, with $\operatorname{dim}(X)=0$. Then, $X$ is compact if and only if $\mathscr{T}_{f}$ is second-countable for every continuous weak selection $f$ for $X$.

Proof: If $X$ is compact and $\mathscr{T}$ is the topology of $X$, then $\mathscr{T}_{f}=\mathscr{T}$ for every continuous weak selection $f$ for $X$. Note that, in this case, $X$ has at least one continuous weak selection because it is a subset of the Cantor set. Suppose that $X$ is not compact. We have the following two possibilities. If $X$ is not separable, then it should have an uncountable discrete cover consisting of open sets because $\operatorname{dim}(X)=0$. On the other hand, it has a continuous weak selection because, for instance, the topology of $X$ is generated by some linear ordering on $X,[\mathbf{8}],[\mathbf{9}]$ (see, also, [2]). Then, by Proposition 2.3, $X$ has a continuous weak selection $g$ such that $\mathscr{T}_{g}$ is not second-countable. In case $X$ is separable, by Theorem 4.1, we get again that $X$ has a continuous weak selection $f$ such that $\mathscr{T}_{f}$ is not second-countable. Thus, if $\mathscr{T}_{f}$ is second-countable for any continuous weak selection for $X$, then $X$ must be compact.

\section{A condition for the existence of continuous weak selections}

In this section, we demonstrate some natural relations between the existence of continuous weak selections, and the Eilenberg's result [1, Theorem I] about ordered topological spaces. In fact, these relations 
were naturally incorporated in Lemma 3.1. Now, we provide another reading of this lemma in terms of Eilenberg's condition of orderability in [1], and some possible further consequences. Following the terminology in $[\mathbf{1}]$, for a space $X$, we let $\Delta(X)=\{(x, x): x \in X\}$, and $P(X)=X^{2} \backslash \Delta(X)$. Also, we consider the map $\Lambda: P(X) \rightarrow P(X)$ defined by $\Lambda(x, y)=(y, x)$, whenever $(x, y) \in P(X)$.

Theorem 5.1. A space $X$ has a continuous weak selection if and only if there are non-empty open subsets $\mathscr{L}$ and $\mathscr{R}$ of $P(X)$ such that $\mathscr{L} \cap \mathscr{R}=\varnothing$, $\mathscr{L} \cup \mathscr{R}=P(X)$, and $\mathscr{R}=\Lambda(\mathscr{L})$.

Proof: Let $f$ be a continuous weak selection for $X$. Following Lemma 3.1, we let $\mathscr{L}=\left\{(x, y) \in P(X): x \prec_{f} y\right\}$ and $\mathscr{R}=\Lambda(\mathscr{L})$, where $\preceq_{f}$ is the selection relation generated by $f$. Then, $\mathscr{L} \cap \mathscr{R}=\varnothing$ and $\mathscr{L} \cup \mathscr{R}=P(X)$. Also, $\mathscr{L}$ is open in $P(X)$ if and only if $\mathscr{R} \subset P(X)$ is open. Hence, Lemma 3.1 completes the proof of this implication.

Suppose now that there are open subsets $\mathscr{L}, \mathscr{R} \subset P(X)$ such that $\mathscr{L} \cap \mathscr{R}=\varnothing, \mathscr{L} \cup \mathscr{R}=P(X)$, and $\mathscr{R}=\Lambda(\mathscr{L})$. Next, define a selection $f:[X]^{2} \rightarrow X$ by letting $f(\{x, y\})=x$ if $(x, y) \in \mathscr{L}$. Note that if $x \neq y$, then either $(x, y) \in \mathscr{L}$ or $(y, x) \in \mathscr{L}$, so our definition is correct. That $f$ is continuous, it follows by Lemma 3.1 .

Corollary 5.2. Let $X$ be a space which has a continuous weak selection. Then, $P(X)$ is not connected.

Proof: According to Theorem 5.1, there are non-empty open subsets $\mathscr{L}, \mathscr{R} \subset P(X)$ such that $\mathscr{L} \cap \mathscr{R}=\varnothing$ and $\mathscr{L} \cup \mathscr{R}=P(X)$. In particular, both $\mathscr{L}$ and $\mathscr{R}$ are clopen in $P(X)$.

Corollary 5.3. Let $X$ be a connected space which has a continuous weak selection. Then, $P(X)$ consists of two components $\mathscr{L}$ and $\mathscr{R}$ such that $\mathscr{R}=\Lambda(\mathscr{L})$.

Proof: By Corollary 5.2, $P(X)$ is not connected. The rest of the proof follows precisely that one in $[\mathbf{1},(3.1)]$.

Corollary 5.4. Let $X$ be a connected space which has a continuous weak selection, and let $E(X)=\{x \in X: X \backslash\{x\}$ is connected $\}$. Then, $|E(X)| \leq 2$.

Proof: By Corollary 5.3, $P(X)$ has exactly two components $\mathscr{L}$ and $\mathscr{R}$ such that $\mathscr{R}=\Lambda(\mathscr{L})$. Suppose that $x, y \in E(X)$ are distinct points, with $(X \backslash\{x\}) \times\{x\} \subset \mathscr{L}$ and $(X \backslash\{y\}) \times\{y\}) \subset \mathscr{L}$. Then, we get that $(y, x) \in \mathscr{L}$ and $(x, y) \in \mathscr{L}$, which is impossible because $\Lambda(x, y)=$ $(y, x)$. Thus, we get that there exists at most one point $x \in E(X)$, with 
$(X \backslash\{x\}) \times\{x\} \subset \mathscr{L}$. In the same way, there exists at most one point $y \in E(X)$, with $(X \backslash\{y\}) \times\{y\} \subset \mathscr{R}$. Hence, $|E(X)| \leq 2$.

Now we get the following result, which, for instance, implies the wellknown fact that the unit circle has no continuous weak selection. Let us recall that we consider only infinite spaces.

Corollary 5.5. Let $X$ be a connected space which has a continuous weak selection. Then, there exists a point $x \in X$ such that $X \backslash\{x\}$ is not connected.

We complete this list of consequences with the following one related to continuous weak selections on product spaces.

Corollary 5.6. Let $X$ be a space such that $X^{2}$ has a continuous weak selection. Then, $X$ must be totally disconnected.

Proof: Let $Z$ be a connected component of $X$, and let us show that $|Z|=1$. Suppose if possible that $|Z| \geq 2$. Since $Z^{2}$ has a continuous weak selection being a subset of $X^{2}$, by Corollary 5.5 , there is a point $(y, z) \in Z^{2}$ such that $Z^{2} \backslash\{(y, z)\}$ is not connected. However, this is not possible because $|Z| \geq 2$ and $Z$ is connected, a contradiction. Thus, any connected component of $X$ is a singleton. Now let us observe that $X$ is naturally embedded in $X^{2}$, hence $X$ has also a continuous weak selection. According to [5, Theorem 4.1], this implies that the connected components of $X$ coincide with the quasi-components of $X$. That is, $X$ is totally disconnected.

\section{References}

[1] S. Eilenberg, Ordered topological spaces, Amer. J. Math. 63 (1941), 39-45.

[2] R. Engelking, "General topology", Translated from the Polish by the author, Second edition, Sigma Series in Pure Mathematics 6, Heldermann Verlag, Berlin, 1989.

[3] S. García-Ferreira, V. Gutev and T. Nogura, Extensions of 2-point selections, New Zealand J. Math. (2006), accepted for publication.

[4] V. Gutev and T. Nogura, Selections and order-like relations, Appl. Gen. Topol. 2(2) (2001), 205-218.

[5] V. Gutev and T. Nogura, Vietoris continuous selections and disconnectedness-like properties, Proc. Amer. Math. Soc. 129(9) (2001), 2809-2815. 
[6] V. Gutev and T. Nogura, Some problems on selections for hyperspace topologies, Appl. Gen. Topol. 5(1) (2004), 71-78.

[7] V. Gutev and T. Nogura, A topology generated by selections, Topology Appl. 153(5-6) (2005), 900-911.

[8] H. HerRlich, Ordnungsfähigkeit total-diskontinuierlicher Räume, Math. Ann. 159 (1965), 77-80.

[9] I. L. Lynn, Linearly orderable spaces, Proc. Amer. Math. Soc. 13 (1962), 454-456.

[10] E. Michael, Topologies on spaces of subsets, Trans. Amer. Math. Soc. 71 (1951), 152-182.

[11] J. van Mill and E. Wattel, Selections and orderability, Proc. Amer. Math. Soc. 83(3) (1981), 601-605.

[12] T. Nogura and D. Shakhmatov, Characterizations of intervals via continuous selections, Rend. Circ. Mat. Palermo (2) 46(2) (1997), 317-328.

[13] P. Urysohn, Zum Metrisationsproblem, Math. Ann. 94(1) (1925), 309-315.

[14] N. Vedenissoff, Remarques sur la dimension des espaces topologiques, (Russian), Uchenye Zapiski Moskov. Gos. Univ. Matematika 30 (1939), 131-140.

Valentin Gutev:

School of Mathematical Sciences

Faculty of Science

University of KwaZulu-Natal

King George V Avenue

Durban 4041

South Africa

E-mail address: gutev@ukzn.ac.za

Artur Tomita:

Departamento de Matemática

Instituto de Matemática e Estatística

Universidade de São Paulo

Caixa Postal 66281

CEP 05315-970 São Paulo

Brazil

E-mail address: tomita@ime.usp.br

Primera versió rebuda el 12 de maig de 2005 ,

darrera versió rebuda el 19 d'abril de 2006. 